MODELING, IDENTIFICATION AND CONTROL, 1993, VOL. 14, NO. 3, 175-180

doi:10.4173/mic.1993.3.5

\title{
A modified LQG algorithm (MLQG) for robust control of nonlinear multivariable systems
}

\author{
JENS G. BALCHEN $\dagger$ \\ Keywords: Multivariable control, robustness, tuning \\ The original LQG algorithm is often characterized for its lack of robustness. This is \\ because in the design of the estimator (Kalman filter) the process disturbance is \\ assumed to be white noise. If the estimator is to give good estimates, the Kalman \\ gain is increased which means that the estimator fails to become robust. A solution \\ to this problem is to replace the proportional Kalman gain matrix by a dynamic PI \\ algorithm and the proportional LQ feedback gain matrix by a PI algorithm. A \\ tuning method is developed which facilitates the tuning of a modified LQG control \\ system (MLQG) by only two tuning parameters.
}

\section{Outline of the MLQG-controller}

In the traditional LQG algorithm which consists of an LQ controller combined with a Kalman filter (Joseph and Tou 1961), it is often observed that the resulting system has a low degree of robustness with respect to stability. Therefore there has been a tendency to discard what is otherwise a very attractive control technique.

The source of this lack of robustness is not to be found in the LQ-control algorithm, but in the estimator which has the structure of a Kalman filter. In the elementary development of a Kalman filter (Kalman and Bucy 1961) the process disturbance is assumed to be white noise. That leads to a proportional Kalman filter gain matrix $(K)$ which will become larger with increasing covariance $(V)$ in the assumed disturbances. In order to achieve a small covariance of the estimation error or, in other words, a small innovation process, one is tempted to increase the assumed covariance of the process disturbance and thereby increase the proportional Kalman filter gain matrix. Thereby the robustness of the estimation loop is drastically reduced since the phase shift near the estimator crossover frequency will change too steeply. Another way to express this is by forcing the process model to follow the process behaviour to very high frequencies so that the innovation process becomes white, the bandwidth of the estimation loop must be excessive and therefore non robust.

But the real disturbances acting upon the process are obviously not white noise sources. In reality it is better to assume that only a smaller portion of the disturbance energy is associated with white noise and the rest at lower frequencies, even zero frequency. This means that a major part of the disturbances may be regarded as slowly varying constants. Thus assuming a zero frequency disturbance, the Kalman filter will have an integrator in its feedback algorithm in parallel with the proportional gain matrix as shown in fig. 1. (Balchen et al. 1973, Balchen 1984).

Received 11 January 1993.

†Department of Engineering Cybernetics, The Norwegian Institute of Technology, 7034 Trondheim. Norway 


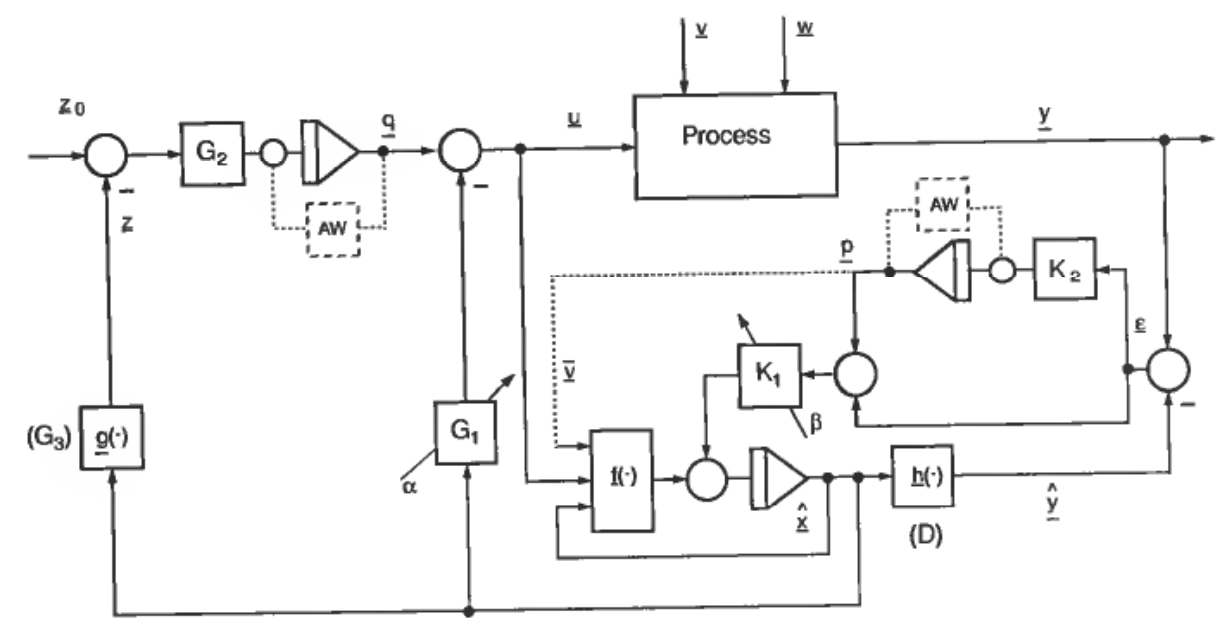

Figure 1. The MLQG Control System.

Consequently the proposed feedback algorithm in the Kalman filter is a proportional plus integral matrix algorithm.

The original LQG algorithm is based on the LQ-algorithm which takes the state estimate $(\hat{\boldsymbol{x}})$ and produces a proportional feedback to the control vector. The result of this feedback is a minimum variance-control system. In most cases of process control, it is however desirable to define a property space $(z)$ which has the same dimension as the control vector space $(u)$ and which expresses the variables which are to be controlled towards certain setpoints. These variables will most often be fewer than those constituting the state space $(x)$, but are related to the state space through a transformation $z=g(x)$.

A multivariable PI algorithm is achieved as shown in Fig. 1 by means of an integral loop controlling the property $(z)$ in parallel with the proportional (LQ) control loop.

Also Fig. 1 shows that both the process model and the measurement model are assumed to be nonlinear. Therefore we are dealing with an extended Kalman filter where all the matrix calculations are based on Jacobian matrices.

The development of the MLQG-controller can be summarized as follows:

Assume the process state space model

$$
\dot{\boldsymbol{x}}=\boldsymbol{f}(\boldsymbol{x}, \boldsymbol{u}, \boldsymbol{v})
$$

with measurements

$$
y=h(x)+w
$$

and properties

$$
z=g(x)
$$

The LQ control is based on the objective function

$$
L(x, u)=x^{\mathrm{T}} Q x+\frac{1}{\alpha} u^{\mathrm{T}} P u
$$

where

$$
Q=\left\{q_{i i}\right\} \geqslant 0 \text { and } q_{i i}=\frac{1}{\Delta x_{i}^{2}}
$$




$$
\begin{aligned}
P & =\left\{p_{i i}\right\}>0 \text { and } p_{i i}=\frac{1}{\overline{\Delta u_{i}^{2}}} \\
\overline{\Delta x_{i}^{2}} & =\text { acceptable variance of } x_{i} \\
\overline{\Delta u_{i}^{2}} & =\text { acceptable variance of } u_{i} \\
\alpha & =\text { tuning parameter }
\end{aligned}
$$

By changing $\alpha$, the magnitudes of the control variables relative to the state variables will be altered. An increase in $\alpha$ will result in an increase in the magnitudes of the control variables. Using elementary optimal control theory and introducing

$$
A=\frac{\partial f(\cdot)}{\partial x}, \quad B=\frac{\partial f(\cdot)}{\partial u}, \quad C=\frac{\partial f(\cdot)}{\partial v}, \quad D=\frac{\partial h(\cdot)}{\partial x}
$$

the Riccati equation will become

$$
\dot{R}=-R A-A^{\mathrm{T}} R+\alpha R B P^{-1} B^{\mathrm{T}} R-Q
$$

and the LQ control gain matrix

$$
G_{1}=\alpha P^{-1} B^{\mathrm{T}} R
$$

Thus tuning $\alpha$ will result in changing the $G_{1}$-matrix.

Similarly the tuning parameter $\beta$ is introduced to modify the assumed white noise component of $(v)$ of the process disturbance relative to the measurement noise $(w)$. Using the notation

$$
\begin{aligned}
& V=\operatorname{cov} \boldsymbol{v}=\left\{\overline{\mathrm{v}_{i}^{2}}\right\} \\
& W=\operatorname{cov} \boldsymbol{w}=\left\{\overline{\mathrm{w}_{i}^{2}}\right\} \\
& X=\operatorname{cov} \boldsymbol{x}=E(\boldsymbol{x}-\hat{\boldsymbol{x}})(\boldsymbol{x}-\hat{\boldsymbol{x}})^{\mathrm{T}}
\end{aligned}
$$

we get

$$
\dot{X}=A X+X A^{\mathrm{T}}+\beta C V C^{\mathrm{T}}-X D^{\mathrm{T}} W^{-1} D X
$$

and the conventional Kalman filter gain matrix

$$
K_{1}=X D^{\mathrm{T}} W^{-1}
$$

Thus we see that by changing the tuning parameter $\beta$ we will have a change in the Kalman filter gain $K_{1}$.

\section{The integral loops}

The integral parallel branches of the control loop and the estimation loop can be designed in a number of ways.

Both integral loops can be designed using extensions of the techniques applied for the proportional loops. For instance in the Kalman filter design it can be assumed that the process disturbance is the sum of two components, one being white noise (as before) and another being the integral of white noise (uncorrelated). Thereby the Kalman filter will contain an integral branch in parallel with the ordinary proportional branch.

The gain $K_{2}$ of the integral branch is computed by an extension of the Riccati equation for the estimation error covariance which is now of $2 n \times 2 n$ dimension instead of $n \times n$ in original system. 
Another, and probable just as logical, way is to prescribe a certain dynamic behaviour of the quantities appearing at the outputs of the integrators namely the vectors $q$ and $p$ indicated in Fig. 1. Inspired from classical SISO PI-controller tuning one can specify the eigenvalues of the integral loops as $\Lambda_{G}$ for the control loop and $\Lambda_{K}$ for the estimation loop.

Since the integral loops will be appreciably slower than the proportional loops, we can arrive at the following differential equations which are good approximations for the two integral loops.

$$
\begin{gathered}
\dot{q}=G_{2} G_{3}\left(A-B G_{1}\right)^{-1} B q=\Lambda_{G} q \\
\dot{p}=K_{2} D\left(A-K_{1} D\right)^{-1} K_{1} p=\Lambda_{K} p
\end{gathered}
$$

where

$$
G_{3}=\frac{\partial g(\cdot)}{\partial x}
$$

These lead to the integral loop gain matrices

$$
G_{2}=\Lambda_{G}\left(G_{3}\left(A-B G_{1}\right)^{-1} B\right)^{-1}
$$

and

$$
K_{2}=\Lambda_{K}\left(D\left(A-K_{1} D\right)^{-1} K_{1}\right)^{-1}
$$

The choice of $\Lambda_{G}$ and $\Lambda_{K}$ may be done as in SISO-systems (Ziegler and Nichols 1942) namely

$$
\Lambda_{G}=k_{1} \Lambda_{\left(A-B G_{1}\right)} \text { or } \lambda_{G i}=-k_{1}\left\|\lambda_{\left(A-B G_{1}\right) i}\right\|
$$

and

$$
\Lambda_{K}=k_{2} \Lambda_{\left(A-K_{1} D\right)} \text { or } \lambda_{k i}=-k_{2}\left\|\lambda_{\left(A-K_{1} D\right) i}\right\|
$$

where $\Lambda_{\left(A-B G_{1}\right)}$ and $\left.\Lambda_{\left(A-K_{1} D\right.}\right)$ are the dominant eigenvalues of the control loop and the estimation loop respectively and the factors $k_{1}$ and $k_{2}$ could be of the order $0 \cdot 1-0 \cdot 2$. Many detailed arguments can be made in this respect.

In Eqn. (9) it is seen that the matrix $G_{3}\left(A-B G_{1}\right)^{-1} B$ must be nonsingular. The choice of the property transformation is essential for the behaviour of the integral control loop. A singular value decomposition of Eqn. (9) will reveal undesirable choices of transformations. Similarly in Eqn. (10) it is necessary that the matrix $D\left(A-K_{1} D\right)^{-1} K_{1}$ is nonsingular which in turn presents restrictions on suitable choices of measurement locations $(D)$ in the process.

When implementing the MLQG strategy it is assumed that all matrix calculations are performed online. That is rather straightforward, but it requires a computational capacity which will increase rapidly with the dimensions of the system. In terms of necessary computer time only cases with fast processes with time constants in the range of seconds will become critical. To increase computational speed it would be beneficial to develop recursive forms of Eqns (9) and (10). The determination of Jacobian matrices such as A, B etc. will mostly be done numerically, but in some cases this can be achieved analytically which will require less computer time.

Open integrators as they appear both in the property control loop and in the estimation have to be equipped with anti-windup precautions (AW) in case their outputs reach amplitude restrictions. In the property control loop this is important because the control vector $(\boldsymbol{u})$ definitely has amplitude constraints given by physical considerations. 
What will happen when such constraints are reached is another matter. Usually nothing but a degradation of the system performance will take place. But since the behaviour of a multivariable feedback system is complicated, undesirable effects may also occur. If an open loop unstable multivariable process has been stabilized by means of feedback the system may again become unstable when one or more control variables reach saturation levels. This particular problem is not dealt with in any detail here because it deserves a much broader treatment than space permits (Balchen 1993).

\section{Consequences of nonlinear modeling}

As is seen in Fig. 1, the estimator contains a nonlinear model of the process. This will be essential in most cases of process control with the rare exception of when a linear model can describe the process behaviour over the entire operating range that is required. The original LQG-algorithm assumes a linearized model around the operating point in the state space, but most often it is just as easy to implement a nonlinear model. Nevertheless the linearized description (Jacobian-matrices) has to be found in order to calculate the feedback matrices both in the control loop and in the estimation loop. The importance of using the nonlinear model in the estimation loop lies in the need to determine the correct operating point. With the inclusion of an integral term in the feedback of the estimator, the expected value of the innovation process $(E(\varepsilon))$ will be forced to zero.

This also causes a problem which has to be taken seriously. In cases when the process disturbance is a parameter variation rather than an additive signal, as assumed in the model structure of Fig. 1, it is evident that the integral action of the estimator may force the process model to erroneous steady state operating conditions.

Care should be taken in modeling the most relevant disturbances properly to avoid such behaviour. From the conditions for observability it is easily derived that the maximal number of disturbances which can be compensated for is $m=$ number of measurements. Also it may be fruitful to regard the quantity $p$ as an estimate of the mean of the process disturbance as indicated by a dotted line in Fig. 1. Furthermore it is necessary to set limits to the integrator output amplitudes by means of anti windupmechanisms.

Another obvious way to tackle the undesirable consequences of modeling errors is to employ a parameter estimation scheme around the nonlinear model. Many methods are available for this purpose, but it is beyond the purpose of this paper to discuss such methods in detail.

\section{Discussion}

The algorithm developed above and shown in Fig. 1 has the following very attractive features:

1. It is based on a state-space model depicting the real behaviour of the process.

2. It takes realistic limitations in the state and control variables into account and considers process disturbances and measurement noise.

3. It only has two tuning parameters which is the same number as with SISO PI-control. This is possible because the a priori knowledge of the relative process excitations and process responses has been utilized.

4. The $\alpha$-parameter adjusts the low and medium frequency behaviour of the control loops whereas the $\beta$-parameter tunes the high frequency rolling off behaviour of the control loops. 


\section{Applications}

Numerous applications of the MLQG have already been suggested and tested.

For the control of the production process for polypropene the MLQG has been shown to give superior performance compared to a number of other control strategies including a variety of conventional PI structures with different pairings of variables 'Lie 1990, Lie and Balchen 1992).

In Jalving, Balchen and Strand (1993), the MLQG has given excellent control of a simulated Fluidized Catalytic Cracker. Conventional multiple PI-control structures with different pairings are known to give unsatisfactory control of this process.

In Amundsen (1992), an MLQG-control system for a rotary kiln is studied yielding very satisfactory results.

Furthermore it is suggested the MLQG-technique is excellent for the control of industrial processes described by models with a large number of state variables relative to the number of control variables e.g. the ferrosilicon process ( $\mathrm{FeSi}$ ) (Valderhaug and Balchen 1992) and the thermomechanical pulping process (TMP) (di Ruscio and Balchen 1992).

\section{Conclusion}

Model based control techniques like the MLQG are attractive for the design of multivariable control systems because they are systematic and logical. With modern inexpensive computer systems the calculations required can be implemented without difficulties.

\section{REFERENCES}

AMUNDSEN, S. (1992). Modeling and control of rotary kiln processes. MSc Thesis at Dept. of Eng. Cybernetics, The Norwegian Inst. of Technology (in Norwegian).

BalChen, J. G., Endresen, T., FJeld, M. and Olsen, T. O. (1973). Multivariable PID estimation and control in systems with biased disturbances. Automatica, 9, 295-307.

BALCHEN, J. G. (1984). A quasi-dynamic optimal control strategy for non-linear multivariable processes based upon non-quadratic objective functionals. Modeling, Identification and Control, 5, 195-209.

BALCHEN, J. G., (1993). Algorithms for the control of multivariable processes with saturating control variables. Modeling, Identification and Control, 14, no. 4 (to appear).

DI Ruscio, D. and BALCHEN, J. G. (1992). A state space model for the TMP process. Considered for inclusion in the Journal of Pulp and Paper Science.

JaLVING, B., BALCHEN, J. G. and Strand, S. (1993). Modified LQG-control and quasi-dynamic optimal control for non-linear multi-variable processes. Paper accepted for IF AC World Congress, Sidney, Australia.

JosEPH, P. D., and TOU, J. (1961). On linear control theory. Trans AIEE (Appl. and Industry), 80, 193-196.

Kalman, R. E., and BuCY, R. S. (1961). New results in linear filtering and prediction theory. Trans. ASME. J. Basic Eng., 83D, 95-108.

LIE, B. (1990). Control structures for polymerization processes applied to polypropene manufacturing. Dr. Ing. thesis, Department of Engineering Cybernetics, The Norwegian Inst. of Technology. Report no:: 1990-82-W.

LIE, B.and BALCHEN, J. G. (1992). A comparison of strategies for the control of a polypropene reactor. Proc. 3rd IFAC Symposium on Dynamics and Control of Chemical Reactors, Distillation Columns and Batch Processes--DYCORD + 92. (Pergamon Press, London).

VALDERHAUG, AA., BALCHEN, J. G. (1992): Modelbased control of the ferrosilicon process. 3rd IFAC Symposium on Dynamics and Control of Chemical Reactors, Distillation Columns and Batch Processes-DYCORD+'92. (Pergamon Press, London).

ZIEGLER, J. G., and NICHOLS, N. B. (1942). Optimum settings of automatic controllers. ASME Transactions, 64, No. 8, p. 759. 